\title{
Analysis of Different Ranges for Wireless Sensor Node Localization using PSO and BBO and its variants
}

\author{
Satvir Singh \\ SBS State Technical Campus \\ Ferozepur, Punjab [INDIA]
}

\author{
Shivangna \\ SBS State Technical Campus \\ Ferozepur, Punjab [INDIA]
}

\author{
Shelja Tayal \\ SBS State Technical Campus \\ Ferozepur, Punjab [INDIA]
}

\begin{abstract}
In a Wireless Sensor Network (WSN) accurate location of target node is highly desirable as it has strong impact on overall performance of the WSN. This paper proposes the application of different migration variants of Biogeography-Based Optimization (BBO) algorithm and Particle Swarm Optimization (PSO) for distributed optimal localization of randomly deployed sensors for different ranges. Biogeography is collective learning of geographical allotment of biological organisms. BBO has a new inclusive vigor based on the science of biogeography and employs migration operator to share information between different habitats, i.e., problem solution. PSO models have only fast convergence but less mature. An investigation on distributed iterative localization is presented in this paper that shows how time consumption and error varies for different ranges. Here the nodes that get localized in iteration act as anchor node. A comparison of the performance of PSO and different migration variants of $\mathrm{BBO}$ in terms of number of nodes localized, localization accuracy and computation time is presented.
\end{abstract}

\section{Keywords:}

Particle Swarm Optimization, Biogeography Based Optimization, Enhanced BBO, Immigration Refusal, Blended BBO, Localization, Wireless Sensor Networks

\section{INTRODUCTION}

WSN is a collection of large number of sensor nodes those are connected wirelessly in an ad-hoc manner [1]. Each node is provided with sensors, transceiver, information processor and power supply, etc. The purpose of WSN is to collect and supply sensed information to a designated sink from a wider area. However, due to size, power supply and constraints the transceiving range is limited and are networked with each other to pass information to the sink. Information received at destination is of use only if the origin of the source, i.e., location of the sensor node is known. Moreover, location of all randomly deployed sensor nodes are also required to determine the route for information passing. Self organizing and fault tolerance characteristics of WSN make them promising for a number of military and civilian applications [2] 3]. To determine the physical coordinates of group of sensor nodes in WSN is one of challenging problem. Some WSN challenges and constraints are Self-Management, Wireless Networking, Design Constraints, Security.

\subsection{Localization}

Localization is most active research area in WSN and it usually refers to the process of determining positions of unknown nodes (target nodes) that uses information of positions of some known nodes i.e., anchor nodes based on measurements such as distance, Time of Arrival (TOA), Time Difference of Arrival (TDOA), Angle of Arrival (AOA), etc. [4, 5, 6]. Many of the applications proposed for WSN require knowledge of sensing information which gives rise to problem of localization. The localization estimation is a two-phase process involving:-

(1) Ranging: Node estimates their distance from anchors (beacons or settled nodes) using signal propagation time or strength of received signal. Precise measurement of these parameters is not possible due to noise; therefore, results of localization algorithms that use these parameter are likely to be inaccurate.

(2) Position Estimation: It is carried out using the ranging information. This is done either by solving a set of simultaneous equations, or by using an optimization algorithm that minimizes the localization error. This is an iterative process, where settled nodes i.e., anchors and localization process is repeated until either all nodes are settled, or no more can be localized [7, 8].

\section{LITERATURE SURVEY}

A survey of localization systems of WSNs is available in [9]. An efficient localization system that extends GPS capabilities to non-GPS nodes in an ad-hoc network as anchors transmit their location information to all nodes in the network is proposed in [10]. Then, each target node estimates its location by performing triangularization. Localization accuracy of node is improved by measuring their distances from their neighbors in [11]. The issue of error accumulation is addressed in [12] through Kalman filter based least square estimation in [13] 14] to simultaneously locate the position of all sensor nodes. Node localization problem is addressed using convex optimization based on semi-definite programming. The semi-definite programming approach is further extended to non-convex inequality constraints in [15]. In [16], Gradient search technique demonstrates the use of data analysis technique called multidimensional scaling (MDS) for estimating the target node positions. WSN is treated as multidimensional optimization problem and addressed through population based stochastic approaches. In [17] centralized location of WSN nodes is proposed by PSO to minimize average localization error. In this approach it provides more accurate localization as compared to simulated annealing algorithm proposed earlier [18]. This approach required few known nodes (anchors) to localize all target nodes. [19] proposes application of BBO and HPSO algorithm for distributed iterative node localization in WSNs. 
Some Genetic Algorithms (GA) based node localization are proposed in [20, 21, 22, 23]. Centralized algorithm determines location of target node by estimating their distances from all one hop neighbors. Each target node is localized under imprecise measurement of distances from three or more neighboring anchors or settled nodes. The method proposed in this paper has following advantages over some of the earlier methods:

(1) Localization is robust against uncertainty of noise associated with distance measurement.

(2) Localization accuracy is better and has fast convergence.

(3) In each iteration, one node gets settled. Thus, each node gets more references in its transmission range. This leads to minimization in error due to flip ambiguity, the situation that arises as reference (anchor) nodes are in non-collinear locations.

This paper proposes two optimization algorithms for distributed iterative node localization in a WSN. The first algorithm is PSO [24] and second is BBO [25] and its variants, i.e., Blended BBO, Immigration Refusal, Enhanced BBO. Variants of BBO have never been proposed for distributed iterative node localization. The rest of the paper is organized as follows: Section 3 explains PSO, BBO, Blended BBO, Immigration Refusal, Enhanced BBO for localization in this study, Section 4 explains how the localization problem is approached using the above mentioned optimization methods, Section 5 discusses numerical simulation and results obtained. At the last, Section 6 presents conclusions and make a projection on possible future research path.

\section{LOCALIZATION METHODS}

The stochastic algorithms PSO, BBO, Blended BBO, EBBO, Refusal $\mathrm{BBO}$ are discussed in the following subsections.

\subsection{Particle Swarm Optimization}

PSO is a robust stochastic optimization technique based on the movement and intelligence of swarms. It was developed in 1995 by James Kennedy (social-psychologist) and Russell Eberhart (electrical engineer). It uses a number of particles that constitute a swarm moving around in the search space looking for the best solution. Each particle keeps track of its coordinates in the solution space which are associated with the best solution (fitness) that has achieved so far by that particle. This value is called personal best, pbest. Another best value that is tracked by the PSO is the best value obtained so far by any particle in the neighborhood of that particle. This value is called gbest. The basic concept of PSO lies in accelerating each particle toward its pbest and the gbest locations, with a random weighted acceleration at each time step as shown in Fig. 1.

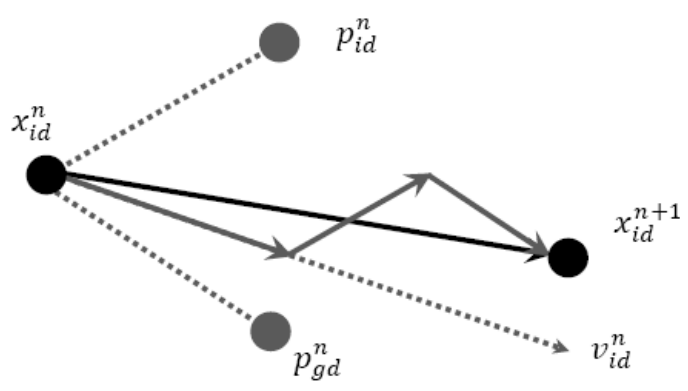

Fig. 1. PSO Characterstics

Consider that the search space is $M$-dimensional and $i$-th particle location in the swarm can be represented by $X_{i}=$ $\left[x_{i 1}, x_{i 2}, \ldots x_{i d} \ldots, x_{i M}\right]$ and its velocity can be represented by

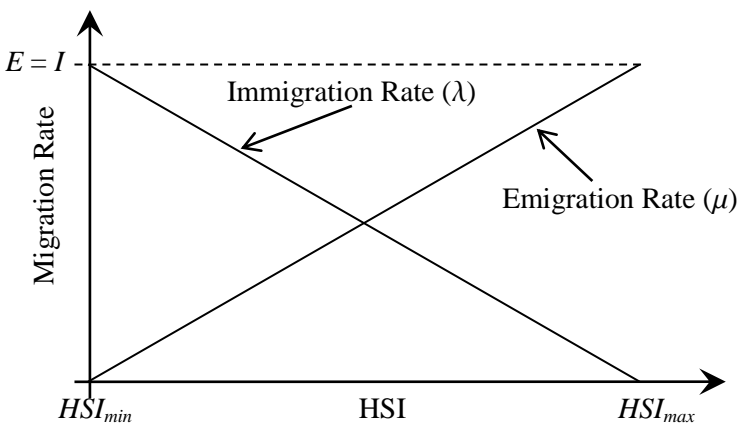

Fig. 2. Migration Curves

another $M$-dimensional vector $V_{i}=\left[v_{i 1}, v_{i 2}, \ldots . v_{i d} . ., v_{i M}\right]$. Let the best previously visited location position of this particle be denoted by $P_{i}=\left[p_{i 1}, p_{i 2}, \ldots p_{i d} . ., p_{i M}\right]$, whereas, $g$-th particle, i.e., $P_{g}=\left[p_{g 1}, p_{g 2}, \ldots . p_{g d} . ., p_{g M}\right]$, is globally best particle location. Fig. 1 depicts the vector movement of particle element from location $x_{i d}^{n}$ to $x_{i d}^{n+1}$ in $(n+1)$-th iteration that is being governed by past best location, $p_{i d}^{n}$, global best location, $p_{g d}^{n}$, and current velocity $v_{i d}^{n}$. Alternatively, the whole swarm is updated according to the equations (1) and (2) suggested by [26], [27].

$$
v_{i d}^{m+1}=\chi\left(w v_{i d}^{m}+\psi_{1} r_{1}\left(p_{i d}^{m}-x_{i d}^{m}\right)+\psi_{2} r_{2}\left(p_{g d}^{m}-x_{i d}^{m}\right)\right)
$$

$$
x_{i d}^{m+1}=x_{i d}^{m}+v_{i d}^{m+1}
$$

Here, $w$ is inertia weight, $\psi_{1}$ is cognitive learning parameter, $\psi_{2}$ is social learning parameter and constriction factor $\chi$, are strategy parameters of PSO algorithm, while $r_{1}$ and $r_{2}$ are random numbers uniformly distributed in the range $[0,1]$.

\subsection{Biogeography-Based Optimization}

BBO is a population based global optimization technique developed on the basis of the science of biogeography, i.e., study of the distribution of animals and plants among different habitats over time and space.

Originally, biogeography was studied by Alfred Wallace [28] and Charles Darwin [29] mainly as descriptive study. However, in 1967, the work carried out by MacAurthur and Wilson [30] changed this view point and proposed a mathematical model for biogeography and made it feasible to predict the number of species in a habitat. For sake of simplicity, it is safe to assume a linear relationship between HSI (or population) and immigration and emigration rates and same maximum emigration and immigration rates, i.e., $E=I$ as depicted graphically in Fig. 2 .

For $k$-th habitat, i.e., $H S I_{k}$, values of emigration rate and immigration rate are given by (3) and (4).

$$
\begin{gathered}
\mu_{k}=E \cdot \frac{H S I_{k}}{H S I_{\max }-H S I_{\min }} \\
\lambda_{k}=I \cdot\left(1-\frac{H S I_{k}}{H S I_{\max }-H S I_{\min }}\right)
\end{gathered}
$$

Algorithmic flow of BBO involves two mechanisms, i.e., migration and mutation, these are discussed in the following subsections.

\subsection{Migration}

Migration is a probabilistic operator that improves HSI of poor habitats by sharing features from good habitats. During Migration, $i$ th habitat, $H_{i}$ where $(i=1,2, \ldots, N P)$ use its immigration rate, $\lambda_{i}$ given by (4), to probabilistically decide whether to 
immigrate or not. In case immigration is selected, then the emigrating habitat, $H_{j}$, is found probabilistically based on emigration rate, $\mu_{j}$ given by (3). The process of migration is completed by copying values of SIVs from $H_{j}$ to $H_{i}$ at random chosen sites, i.e., $H_{i}(S I V) \leftarrow H_{j}(S I V)$. Migration variants are discussed in the following sections:

3.3.1 Immigration Refusal. In BBO, if a habitat has high emigration rate, i.e, the probability of emigrating to other habitats is high and the probability of immigration from other habitats is low. This BBO variants with conditional migration is termed as Immigration Refusal [31].

3.3.2 Blended Migration. In blended migration, a solution feature of solution ImHbt is not simply replaced by a feature from solution EmHbt as happened in standard BBO migration operator. Instead, a new solution feature, $\operatorname{ImHbt}(S I V)$, solution is comprised of two components, i.e., $\operatorname{ImHbt}(S I V) \leftarrow \alpha$. $\operatorname{ImHbt}(S I V)+(1-\alpha) \cdot E m H b t(S I V)$. Where $\alpha$ is a random number between 0 and 1 .

3.3.3 Enhanced Biogeography Based Optimization. Standard BBO migration operator creates the duplicate solutions which decreases the diversity of the algorithm. To prevent diversity decrease in the population, duplicate habitats are replaced with randomly generated habitats that increases the exploration ability.

\subsection{Mutation}

Mutation is another probabilistic operator that modifies the values of some randomly selected SIVs of every habitat that is intended for exploration of search space for better solutions by increasing the biological diversity in the population. The mutation rate, $m$ Rate, for $k$-th habitats is calculated as (5)

$$
\text { mate }_{k}=C \times \min \left(\mu_{k}, \lambda_{k}\right)
$$

where $N P$ is total number of habitats sorted in ascending order. $E$ and $I$ are maximum emigration and immigration rates, usually $E=I$ and $C$ is a constant and equal to 1 .

\section{STEPS FOLLOWED FOR LOCALIZATION}

The objective of WSN localization is to determine maximum number of $N$ target nodes by using $M$ anchor nodes which know their locations by the process followed:-

(1) $N$ target nodes and $M$ anchor nodes are randomly deployed in a 2-Dimensional sensor field. Each target node and anchor node has a transmission range $R$. At each iteration one node gets settled and works as anchor node in the next iteration and transmits information as the anchors do.

(2) Target node which has atleast 3 anchor nodes in its transmission range is said to be localized.

(3) Mean of coordinates of anchor nodes fall within transmission range, i.e., mean $\left(x_{1}, x_{2}, \ldots . x_{5} \ldots, x_{n}\right)$, mean $\left(y_{1}, y_{2}, \ldots . y_{5} \ldots, y_{n}\right)$ is termed as centroid position.

(4) Randomly deploy few nodes around estimated position and distance between nodes in deployment and anchor nodes in the transmission range are calculated. The distance measurement are effected with gaussian additive noise. A node estimates its distance from anchor i as $\hat{d}_{i}=d_{i}+\eta_{i}$. Where $d_{i}$ is the actual distance and given by following equation

$$
d_{i}=\sqrt{\left(x-x_{i}\right)^{2}+\left(y-y_{i}\right)^{2}}
$$

where $(x, y)$ is the location of target node and $\left(x_{i}, y_{i}\right)$ is the location of $i$-th anchor node in neighborhood of target node. The measurement noise $\eta_{i}$ has a random value which is uniformly distributed in the range $d_{i} \pm d_{i} \frac{\left(P_{n}\right)}{100}$ where $P_{n}$ is percentage noise in distance measurement.
(5) Five case studies are conducted. Each localization target node runs PSO, BBO, Blended BBO, EBBO and Immigration Refusal to localize itself. The objective function is to minimize the average localization error between measured distance and estimated distance. It is defined as follows

$$
f(x, y)=\frac{1}{M} \sum_{i=1}^{M}\left(\sqrt{\left(x-x_{i}\right)^{2}+\left(y-y_{i}\right)^{2}}-\hat{d}_{i}\right)^{2}
$$

where $M \geq 3$ is the number of anchor nodes within transmission range $\mathrm{R}$, of target node.

(6) When all the $N_{l}$ localizable nodes determine their coordinates, total average localization error is calculated as the mean of square of distances of estimated node coordinates $\left(x_{i}, y_{i}\right)$ and the actual node coordinates $\left(X_{i}, Y_{i}\right)$, for $i=$ $1,2,3 \ldots N_{l}$, determines for all cases of PSO, BBO, Blended $\mathrm{BBO}, \mathrm{EBBO}$, Immigration Refusal in following equation

$$
E_{l}=\frac{1}{N_{l}} \sum_{i=1}^{M}\left(\left(x_{i}-X_{i}\right)^{2}+\left(y_{i}-Y_{i}\right)^{2}\right)
$$

(7) Steps 2 to 6 are repeated until all target nodes get localized. The performance of localization algorithm is based on $E_{l}$ and $N_{N l}$, where $N_{N l}=N-N_{l}$ is number of nodes that could not be localized. The minimum the values of $E_{l}$ and $N_{N l}$, the better will be the performance.

\section{SIMULATION RESULTS}

WSN localization simulations and its performance evaluation were conducted using PSO, BBO, Blended BBO, EBBO, Immigration Refusal in $\mathrm{C} / \mathrm{C}++$ environment. Common strategic settings for each case are: (1) Maximum number iterations $=20$ (2) Population size $=10$, (3) Number of target nodes $=50$, (4) Number of anchor nodes $=10$ (5) Transmission range of each node $=20$ and 15 respectively. These target and anchor nodes are randomly deployed in 2-dimensional sensor field having dimensions of $100 \times 100$ square units. In Fig. 3 to Fig. 12, $\nabla$ defines node localization estimated by PSO, BBO, Blended BBO, EBBO and Immigration Refusal respectively, $*$ defines location of node, $\bullet$ defines non-localized nodes and remaining defines the location of anchor nodes.

\subsection{Localization using PSO}

In this case study, each target node that can be localized, runs PSO algorithm to localize itself. The parameters of PSO are set as follows.

(1) Acceleration constants $c_{1}=c_{2}=2.0$

(2) Limits on particle position: $X_{\min }=0$ and $X_{\max }=100$

25 trial experiments of PSO-based localization are conducted for $P_{n}=2$ and $P_{n}=5$ for range 20 and 15 respectively. Average of total localization error $E_{l}$ defined in (8) is computed and shown in Fig. 3 and Fig. 8.

\subsection{Localization using BBO}

In this case study, each target node that can be localized, runs $\mathrm{BBO}$ algorithm to localize itself. The parameters of BBO are set as follows.

(1) Limits on particle position: $X_{\min }=0$ and $X_{\max }=100$

(2) $w=0.01$

25 trial experiments of localization using BBO are conducted for $P_{n}=2$ and $P_{n}=5$ for range 20 and 15 . Average of total localization error $E_{l}$ defined in (8) is computed and shown in Fig. 4 and Fig. 9. The parameters are similar for Fig. 5 to Fig. 7 and Fig. 10 to Fig. 12, i.e., Blended BBO, EBBO, Immigration Refusal, respectively. 


\subsection{Discussions on Results}

The actual locations of nodes and anchors, and the coordinates of the nodes estimated by PSO, BBO, Blended BBO, EBBO, Immigration Refusal in a trail run are shown in Fig. 3 - Fig. 12. The best results are summarized in Table 1 and Table 2 and it can be observed that all stochastic algorithms used here have performed fairly well in WSN localization.

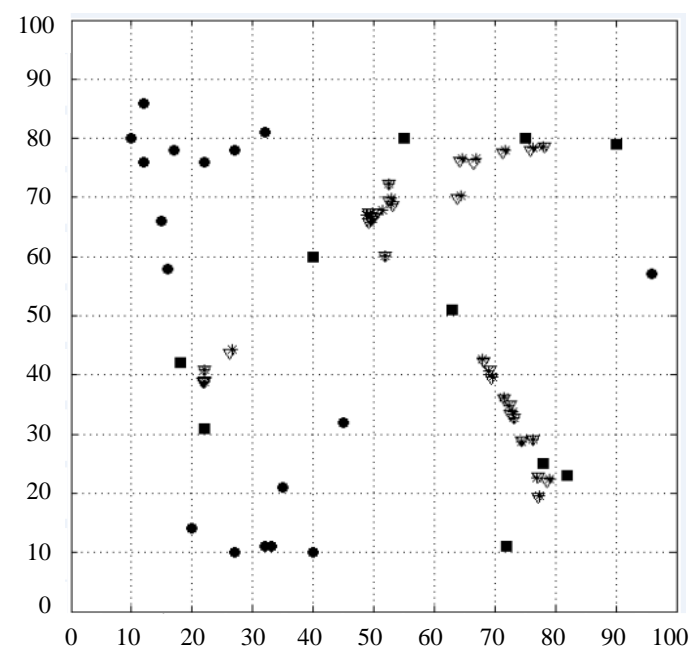

Fig. 3. Location estimated by PSO for Range $=20$

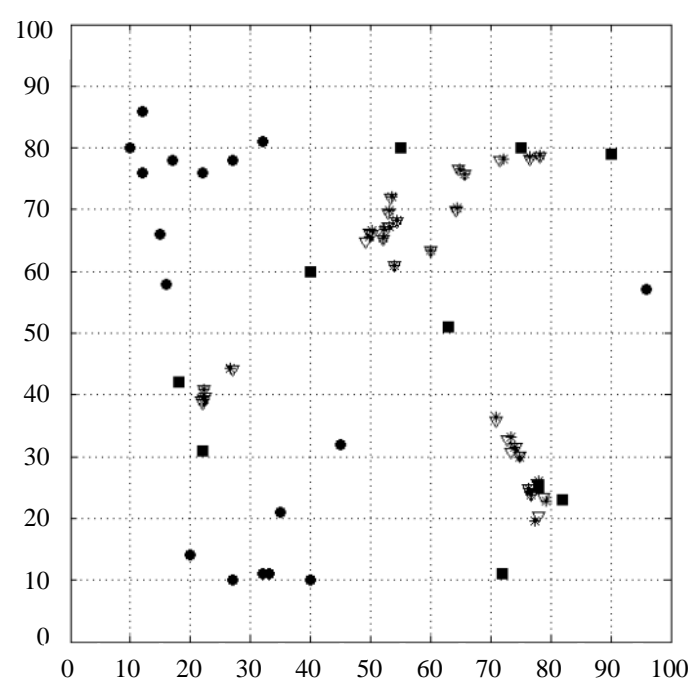

Fig. 4. Location estimated by $\mathrm{BBO}$ for Range $=20$

Average localization error in all algorithms is increased when $P_{n}$ is changed from 2 to 5. Performance of $E_{l}$ for Blended BBO is less as compared to all other algorithms that has been discussed. However the computing time required for Blended $\mathrm{BBO}$ is more as compared to BBO, EBBO, Immigration Refusal. A choice between algorithms influenced by how accurate the localization is expected to be and fast convergence.

\section{CONCLUSION}

Artificial intelligence based single-hop distributed node localization algorithms by PSO, BBO, Blended BBO, EBBO, Immigration refusal have been presented in distributed and iterative fashion. The proposed algorithms have better accuracy and fast convergence. The paper has briefly outlined the algorithms and pre-

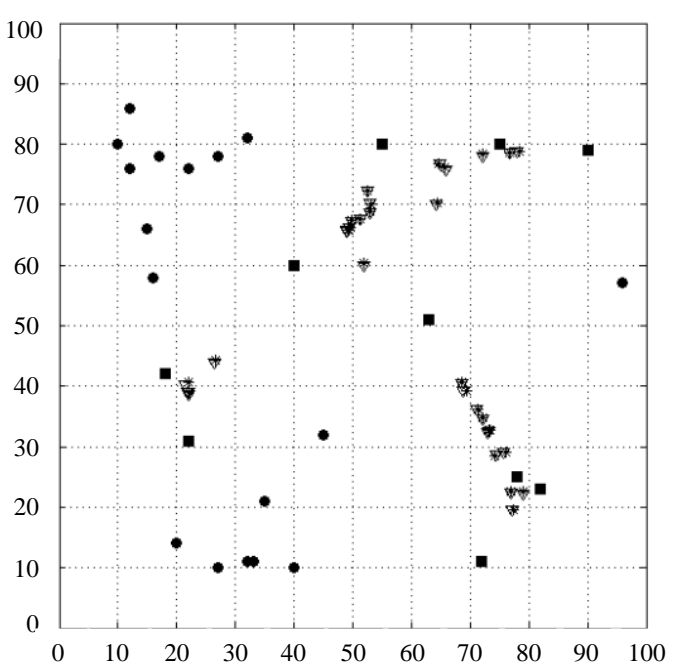

Fig. 5. Location estimated by Blended $\mathrm{BBO}$ for Range $=20$

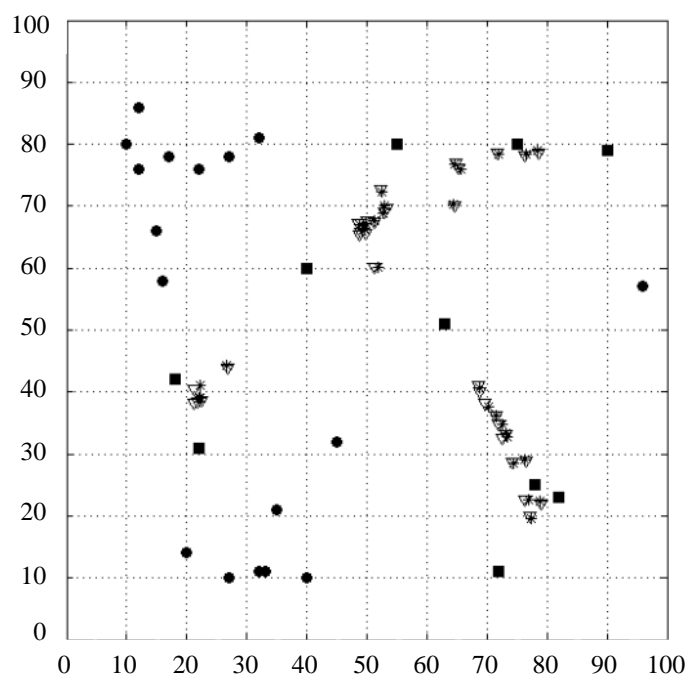

Fig. 6. Location estimated by Enhanced BBO for Range=20

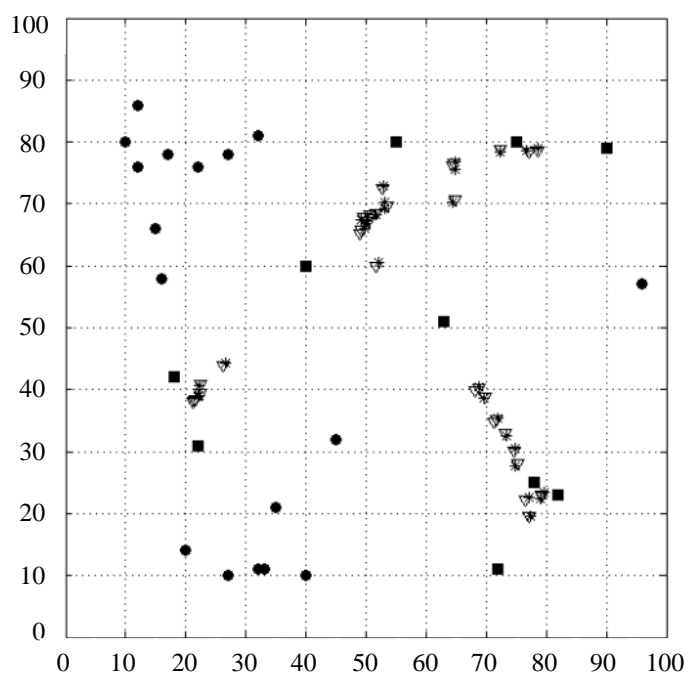

Fig. 7. Location estimated by Immigration Refusal for Range 20 


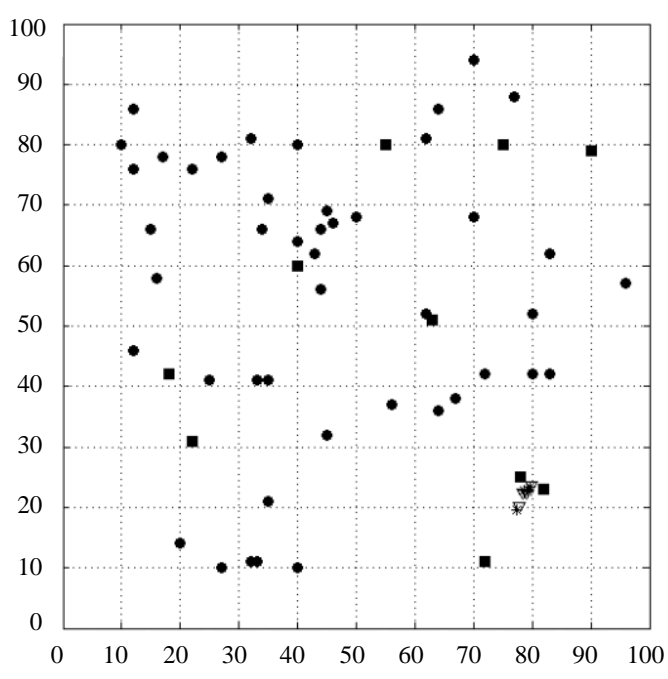

Fig. 8. Location estimated by PSO for Range=15

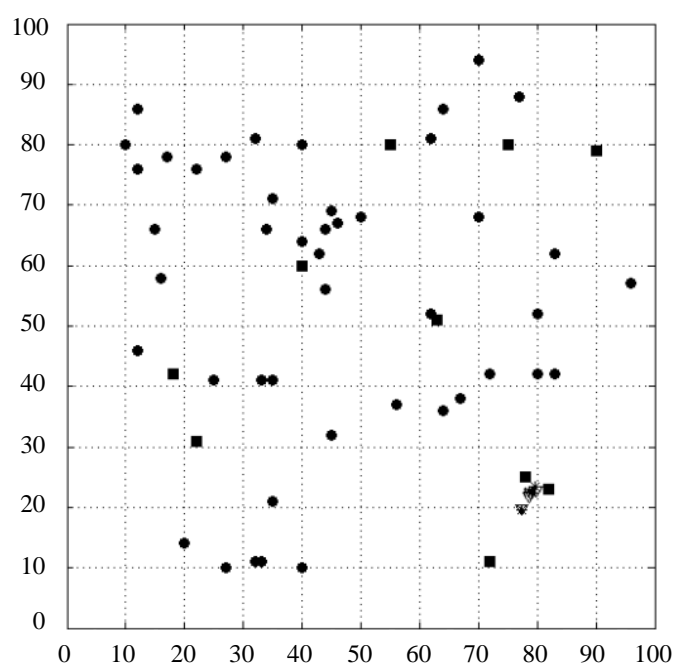

Fig. 9. Location estimated by $\mathrm{BBO}$ for Range $=15$

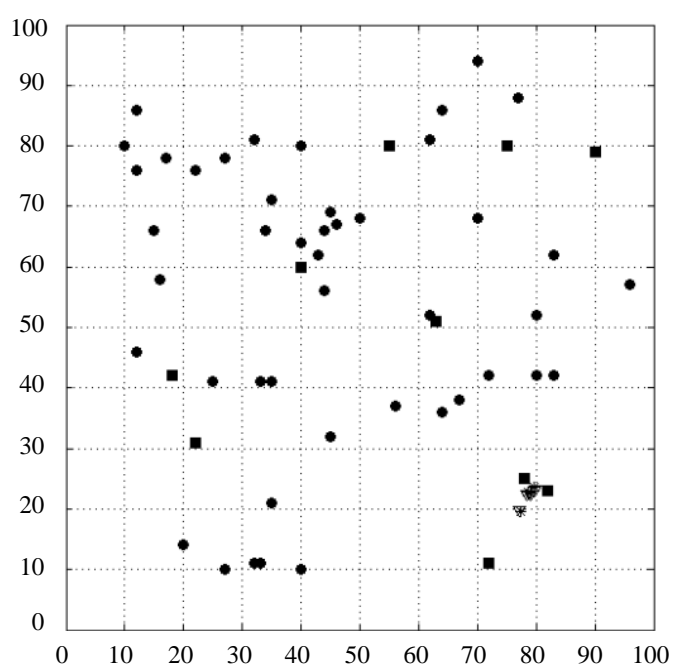

Fig. 10. Location estimated by Blended BBO for Range=15

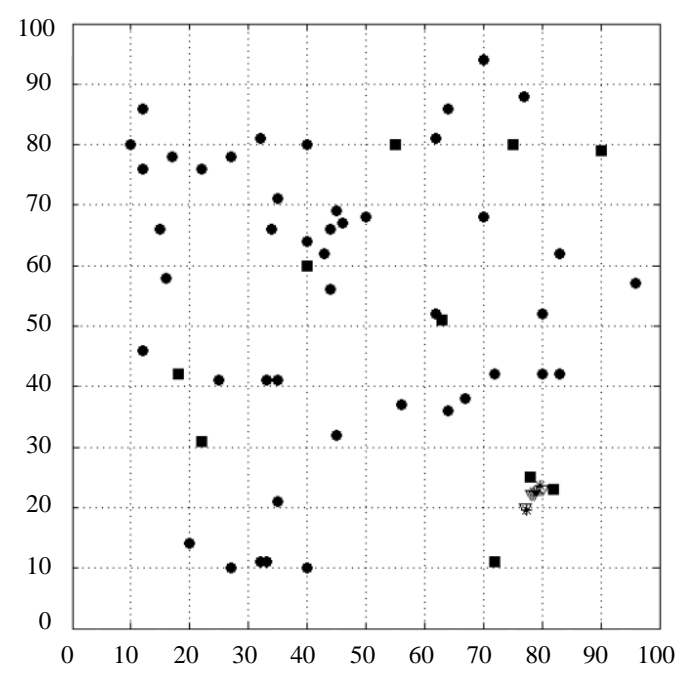

Fig. 11. Location estimated by Enhanced BBO for Range=15

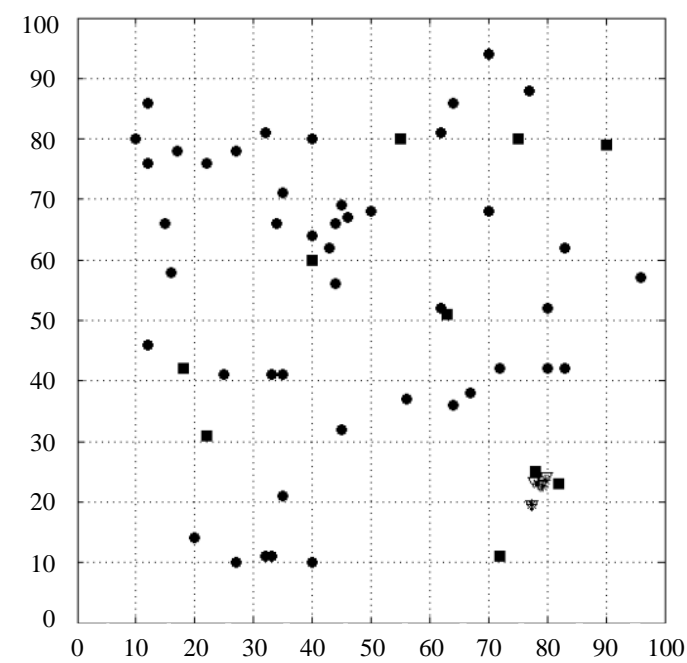

Fig. 12. Location estimated by Immigration Refusal for Range=15

sented a summary of their results for comparison. Blended BBO determines accurate coordinates quickly for both ranges 20 and 15 but the error for range 20 is less as compared to range 15 and time consumed is more for range 20 as compared to range 15. Further Stochastic algorithms can be used in centralized localization method in order to compare performance of centralized and distributed localization methods to minimize average localization error. A choice between the algorithms depends on desired localization speed and accuracy.

Table 1. Summary of 25 trial runs of PSO, BBO, and its variants for Range $=20$

\begin{tabular}{|c|c|c|c|c|}
\hline & \multicolumn{2}{|c|}{$\mathbf{P}_{\mathbf{n}}=\mathbf{2}$} & \multicolumn{2}{c|}{$\mathbf{P}_{\mathbf{n}}=\mathbf{5}$} \\
\hline EAs & $\mathbf{E}_{\mathbf{l}}$ & Time(s) & $\mathbf{E}_{\mathbf{l}}$ & Time(s) \\
\hline PSO & 0.4839 & 0.620 & 0.5777 & 0.618 \\
\hline BBO & 0.5361 & 0.484 & 0.6692 & 0.547 \\
\hline Blended BBO & $\mathbf{0 . 2 5 6 4}$ & 0.502 & $\mathbf{0 . 3 7 2 5}$ & 0.438 \\
\hline EBBO & 0.5877 & 0.469 & 0.6594 & 0.508 \\
\hline Refusal BBO & 0.6204 & 0.556 & 0.7983 & 0.518 \\
\hline
\end{tabular}


Table 2. Summary of 25 trial runs of PSO, BBO, and its variants for Range $=15$

\begin{tabular}{|c|c|c|c|c|}
\hline & \multicolumn{2}{|c|}{$\mathbf{P}_{\mathbf{n}}=\mathbf{2}$} & \multicolumn{2}{c|}{$\mathbf{P}_{\mathbf{n}}=\mathbf{5}$} \\
\hline EAs & $\mathbf{E}_{\mathbf{l}}$ & Time(s) & $\mathbf{E}_{\mathbf{l}}$ & Time(s) \\
\hline PSO & 0.5486 & 0.060 & 0.6133 & 0.073 \\
\hline BBO & 0.6403 & 0.075 & 0.8318 & 0.052 \\
\hline Blended BBO & $\mathbf{0 . 3 4 4 0}$ & 0.069 & $\mathbf{0 . 4 0 0 5}$ & 0.068 \\
\hline EBBO & 0.6219 & 0.067 & 0.7002 & 0.070 \\
\hline Refusal BBO & 0.7107 & 0.053 & 0.7237 & 0.067 \\
\hline
\end{tabular}

\section{REFERENCES}

[1] I. Akyildiz, W. Su, Y. Sankarasubramaniam, and E. Cayirci, "A survey on sensor networks," vol. 40, no. 8. IEEE, 2002, pp. 102-114.

[2] D. Estrin, D. Culler, K. Pister, and G. Sukhatme, "Connecting the Physical World with Pervasive Networks," vol. 1, no. 1. IEEE, 2002, pp. 59-69.

[3] G. Pottie and W. Kaiser, "Wireless integrated network sensors," vol. 43, no. 5. ACM, 2000, pp. 51-58.

[4] L. Doherty, L. El Ghaoui et al., "Convex position estimation in wireless sensor networks," in INFOCOM 2001. Twentieth Annual Joint Conference of the IEEE Computer and Communications Societies. Proceedings. IEEE, vol. 3. IEEE, 2001, pp. 1655-1663.

[5] R. Kulkarni, G. Venayagamoorthy, and M. Cheng, "Bioinspired node localization in wireless sensor networks," in Systems, Man and Cybernetics, 2009. SMC 2009. IEEE International Conference on. IEEE, 2009, pp. 205-210.

[6] A. Pal, "Localization algorithms in wireless sensor networks: Current approaches and future challenges," vol. 2, no. 1, 2010, pp. 45-73.

[7] G. Mao and B. Fidan, "Introduction to wireless sensor network localization," 2009.

[8] N. Patwari, J. Ash, S. Kyperountas, A. Hero III, R. Moses, and N. Correal, "Locating the nodes: Cooperative localization in wireless sensor networks," vol. 22, no. $4 . \quad$ IEEE, 2005, pp. 54-69.

[9] A. Boukerche, H. Oliveira, E. Nakamura, and A. Loureiro, "Localization systems for wireless sensor networks," vol. 14, no. 6. IEEE, 2007, pp. 6-12.

[10] D. Niculescu and B. Nath, "Ad hoc positioning system (aps)," in Global Telecommunications Conference, 2001. GLOBECOM'01. IEEE, vol. 5. IEEE, 2001, pp. 29262931.

[11] C. Rabaey and K. Langendoen, "Robust positioning algorithms for distributed ad-hoc wireless sensor networks," in USENIX technical annual conference, 2002.

[12] A. Savvides, H. Park, and M. Srivastava, "The bits and flops of the n-hop multilateration primitive for node localization problems," in Proceedings of the 1st ACM international workshop on Wireless sensor networks and applications. ACM, 2002, pp. 112-121.

[13] M. Di Rocco and F. Pascucci, "Sensor network localisation using distributed extended kalman filter," in Advanced intelligent mechatronics, 2007 IEEE/ASME international conference on. IEEE, 2007, pp. 1-6.

[14] R. E. Kalman, "A New Approach to Linear Filtering and Prediction Problems," Journal of Basic Engineering, vol. 82, no. 1, pp. 35-45, 1960.
[15] P. Biswas, T. Lian, T. Wang, and Y. Ye, "Semidefinite programming based algorithms for sensor network localization," vol. 2, no. 2. ACM, 2006, pp. 188-220.

[16] T. Liang, T. Wang, and Y. Ye, "A gradient search method to round the semidefinite programming relaxation solution for ad hoc wireless sensor network localization," vol. 5, 2004.

[17] A. Gopakumar and L. Jacob, "Localization in wireless sensor networks using particle swarm optimization," in Wireless, Mobile and Multimedia Networks, 2008. IET International Conference on. IET, 2008, pp. 227-230.

[18] A. Kannan, G. Mao, and B. Vucetic, "Simulated annealing based localization in wireless sensor network," in $L o$ cal Computer Networks, 2005. 30th Anniversary. The IEEE Conference on. IEEE, 2005, pp. 2-pp.

[19] A. Kumar, A. Khosla, J. Saini, and S. Singh, "Computational intelligence based algorithm for node localization in wireless sensor networks," in Intelligent Systems (IS), 2012 6th IEEE International Conference. IEEE, 2012, pp. 431438.

[20] G. Nan, M. Li, and J. Li, "Estimation of node localization with a real-coded genetic algorithm in wsns," in Machine Learning and Cybernetics, 2007 International Conference on, vol. 2. IEEE, 2007, pp. 873-878.

[21] S. Yun, J. Lee, W. Chung, E. Kim, and S. Kim, "A soft computing approach to localization in wireless sensor networks," vol. 36, no. 4. Elsevier, 2009, pp. 7552-7561.

[22] Q. Zhang, J. Wang, C. Jin, and Q. Zeng, "Localization algorithm for wireless sensor network based on genetic simulated annealing algorithm," in Wireless Communications, Networking and Mobile Computing, 2008. WiCOM'08. 4th International Conference on. IEEE, 2008, pp. 1-5.

[23] Q. Zhang, J. Huang, J. Wang, C. Jin, J. Ye, and W. Zhang, "A new centralized localization algorithm for wireless sensor network," in Communications and Networking in China, 2008. ChinaCom 2008. Third International Conference on. IEEE, 2008, pp. 625-629.

[24] J. Kennedy and R. Eberhart, "Particle swarm optimization," in Neural Networks, 1995. Proceedings., IEEE International Conference on, vol. 4. IEEE, 1995, pp. 1942-1948.

[25] D. Simon, "Biogeography-based optimization," vol. 12, no. 6. IEEE, 2008, pp. 702-713.

[26] X. Hu, Y. Shi, and R. Eberhart, "Recent advances in particle swarm," in Evolutionary Computation, 2004. CEC2004. Congress on, vol. 1. IEEE, 2004, pp. 90-97.

[27] Y. del Valle, G. Venayagamoorthy, S. Mohagheghi, J. Hernandez, and R. Harley, "Particle swarm optimization: Basic concepts, variants and applications in power systems," vol. 12, no. 2. IEEE, 2008, pp. 171-195.

[28] A. Wallace, "The geographical distribution of animals," 1876.

[29] C. Darwin and G. Beer, The Origin of Species. Books, Incorporated, 1869.

[30] R. MacArthur and E. Wilson, The Theory of Island Biogeography. Princeton University Press, 2001, vol. 1.

[31] D. Du, D. Simon, and M. Ergezer, "Biogeography-based optimization combined with evolutionary strategy and immigration refusal," in Systems, Man and Cybernetics, 2009. SMC 2009. IEEE International Conference on. IEEE, 2009, pp. 997-1002. 